\title{
Risk of Mycoplasma bovis transmission from contaminated sand bedding to naive dairy calves
}

\author{
D. J. Wilson, ${ }^{\star 1}$ A. Justice-Allen,† G. Goodell,ł T. J. Baldwin, ${ }^{\star}$ R. T. Skirpstunas, ${ }^{\star}$ and K. B. Cavender \\ *Department of Animal, Dairy and Veterinary Sciences, Utah State University, Logan 84321 \\ †Arizona Game and Fish Department, Phoenix 85086 \\ ¥The Dairy Authority, Greeley, CO 80634
}

\section{ABSTRACT}

The objective of this study was to evaluate the possible transmission of Mycoplasma bovis from positive sand bedding to naïve dairy calves. Twelve preweaned Holstein bull calves were blocked in pairs and randomly assigned as unexposed controls $(\mathrm{n}=6)$ bedded with control sand, or exposed calves $(n=6)$ bedded with sand previously positive for $M$. bovis at a dairy farm. Bedding sand was cultured weekly. Nasal and ear swabs and sera were collected weekly, tracheal swabs were collected monthly, and by the end of the 105-d study, all calves were euthanized $(n=10)$ or died $(n=2)$. Sera were tested for M. bovis-specific antibody. Mycoplasma spp. culture was performed on nasal and ear swabs; culture and a PCR differentiating multiple Mycoplasma spp. were performed on postmortem samples of lung, retropharyngeal lymph node, and trachea from each calf. A complete necropsy also was performed. During $6 \mathrm{wk}$, mycoplasma concentration in exposed group sand was between 200 and $32,000 \mathrm{cfu} / \mathrm{g}$. All 166 tracheal swabs, nasal and ear swabs, and postmortem tests from all calves were negative for mycoplasma. All 94 sera were negative for $M$. bovis-specific antibody. No gross pathology suggestive of mycoplasma disease was detected. The probability of mycoplasma detection, if an exposed calf had become infected 4 wk after exposure, ranged between 97 and $99 \%$ depending on time of exposure for individual calves. There was no evidence that sand bedding contaminated with $M$. bovis might serve as a source of transmission to naïve dairy calves.

Key words: bedding, dairy cattle, environment, mycoplasma

\section{INTRODUCTION}

Mycoplasma spp. can infect all ages of cattle, and may cause arthritis, pneumonia, septicemia, and death.

Received August 6, 2010.

Accepted November 16, 2010.

${ }^{1}$ Corresponding author: david.wilson@usu.edu
Adult cows also may contract mastitis, metritis, or agalactia. The most common mycoplasma affecting cattle is Mycoplasma bovis; infections also can be caused by Mycoplasma californicum, Mycoplasma arginini, Mycoplasma canadense, Mycoplasma bovigenitalium, and other Mycoplasma spp. (Pfützner and Sachse, 1996; González and Wilson, 1997; Byrne et al., 2001). Because standard microbial culture methods do not isolate Mycoplasma spp., special laboratory methods are required for diagnosis (Wilson et al., 1992; González et al., 1995; González and Wilson, 1997).

Outbreaks of mycoplasma mastitis and pneumonia have been associated with the introduction of new and presumably infected animals into dairy herds, as well as the recrudescence of infection in asymptomatic carriers. Previously described modes of transmission are direct from animal to animal by inhalation and respiratory secretions and also at milking time via contaminated inflations in milking units (Jasper, 1977; González et al., 1993; Ghazaei, 2006; Wilson et al., 2007).

As part of a follow-up to a mycoplasma mastitis prevalence study in Utah (Wilson et al., 2009), M. bovis was found in multiple samples of recycled sand bedding on a dairy farm during an outbreak of clinical mastitis caused by mycoplasma; diagnosis included culture and confirmation by PCR (Justice-Allen et al., 2010). Some of the sand was transported to Utah State University for long-term study, the results of which were reported previously (Justice-Allen et al., 2010). The objective of this study was to test whether exposure of naïve, preweaned dairy calves to $M$. bovis-positive sand bedding was associated with mycoplasma infection.

\section{MATERIALS AND METHODS}

Holstein bull calves $(\mathrm{n}=12)$ from a few days to $2 \mathrm{wk}$ old from a farm with no evidence of mycoplasma in any cows or calves were sought. Mycoplasma among dairy calves is common; the percentage of dairy herds with at least 1 calf that has positive results for microbial culture of nasal swabs for Mycoplasma spp. has been reported between 80 and 92\% (Springer et al., 1982; 
ter Laak et al., 1992). Prevalence of Mycoplasma spp. ( $M$. bovis is the single most common species isolated) among young dairy calves from 3 to $60 \mathrm{~d}$ old has been reported as greater than 50\% (Muenster et al., 1979; Catry et al., 2008). The sensitivity of diagnostic methods for detection of mycoplasma in an infected calf has been reported as approximately $25 \%$ for nasal or ear swabs (Thomas et al., 2002) and $75 \%$ for tracheal swabs (Marois et al., 2007). Therefore, some assumptions were made to screen for a mycoplasma-free herd. Multiple dairy farms were visited, and 8 calves per farm from 7 to $60 \mathrm{~d}$ old were sampled using nasal and ear swabs. Sensitivity of mycoplasma detection was estimated as 25\%, and mycoplasma prevalence (if the herd had any mycoplasma infections) was estimated at $50 \%$ of the calves; therefore, each calf tested was assumed to have a $12.5 \%$ probability $(0.25 \times 0.50)$ of testing positive for Mycoplasma spp. if the herd was positive. The probability of failure to detect the disease at the herd level in any given calf was $(1-0.125)=0.875$; using sequential probability, the probability of failing to detect mycoplasma in a positive herd (false negative herd status) if 8 of 8 calves tested negative was $(0.875)^{8}=0.34$ (i.e., a $66 \%$ probability that the herd was truly free of mycoplasma in calves). A dairy farm was eventually found where all 8 calves tested negative; the farm was revisited and 24 additional young calves were nasal and ear swabbed for mycoplasma and also all tested negative. With all 32 calves testing negative, the probability that the herd was truly infected and all mycoplasma test results were false-negative was $(0.875)^{32}=0.013$ (i.e., a $99 \%$ probability that the herd was truly free of mycoplasma in calves). After selection of the source herd, 12 additional bull calves were obtained for study on d $1(\mathrm{n}=7), 31(\mathrm{n}=3)$, and 38 $(\mathrm{n}=2)$ of the study based on availability and physical examination by 2 of the authors (D. Wilson and A. Justice-Allen). The study protocol was approved by the Utah State University Institutional Animal Care and Use Committee.

\section{Housing and Feeding of Calves}

Calves were housed in individual plastic hutches with wire fences during the 105-d study. Hutches were approximately $9 \mathrm{ft}(3 \mathrm{~m})$ apart, except that the control calves were separated from the mycoplasma-exposed calves by 80 feet $(25 \mathrm{~m})$. Control calves were bedded at least twice every day with mycoplasma-negative sand from a sand quarry, on top of straw. Exposed calves were bedded at least twice every day with sand previously identified as $M$. bovis-positive (Justice-Allen et al., 2010) on a dairy farm, on top of straw. The straw was added as needed for moisture absorption and com- fort (more frequently in cold or wet weather); sand was added at least twice daily to maximize potential exposure of the exposed group to mycoplasma. The positive sand $(18,000 \mathrm{~kg})$ had been transported to Utah State University and was stored outdoors in a pile for $18 \mathrm{mo}$ before the study reported here began. In a previous study, when the same sand pile was culture-positive for mycoplasma, the dose of M. bovis was always $>200$ cfu/g and usually ranged between 7,800 and 2,130,000 cfu/g (Justice-Allen et al., 2010).

Calves were fed a commercial $20 \%$ CP, $20 \%$ fat allmilk milk replacer in nipple bottles or buckets and a calf starter feed (pelleted grain and alfalfa mix) ad libitum in buckets. Water was provided free choice in buckets. All buckets and other feeding equipment were thoroughly cleaned with soap and water twice daily.

Calves were fed and cared for at every visit beginning with the control calves and then moving to the exposed calves. Boots and disposable gloves were washed and disinfected between every calf. Disposable gloves were changed after handling any visibly sick calf and between the control and exposed groups. Laundered barn clothes were worn to the calf housing area at every visit; no dirty clothes were reused between visits to avoid spread of organisms from the mycoplasma-exposed area to the control area.

\section{Randomized Blocking and Antemortem Diagnostic Sample Collection}

Calves were blocked in pairs by weight and height and randomly assigned as unexposed controls $(\mathrm{n}=6)$ or exposed $(\mathrm{n}=6)$. Blood from jugular venipuncture was collected weekly. Nasal and ear swabs also were collected weekly, except during weeks when tracheal swabs were collected and the week of 1 holiday; tracheal swabs were collected every 4 wk. Serum was harvested from each blood sample. Sera were held at $-20^{\circ} \mathrm{C}$ and shipped in 2 batches, 1 after $10 \mathrm{wk}$ and the remaining samples at the end of the 15-wk study to the Texas Veterinary Medical Diagnostic Laboratory, where they were tested for $M$. bovis-specific antibody using a commercial ELISA, Mycoplasma bovis antibody test kit (Mycoplasma bovis 32; Biovet, Inc., Quebec City, QB, Canada). For tracheal swab collection, calves were anesthetized with i.v. xylazine (Rompun; Bayer Animal Health, Shawnee Mission, KS); a beginning dose of 0.05 $\mathrm{mg} / \mathrm{kg}$ was i.v. administered slowly in the jugular vein and repeated if necessary until recumbency and slack jaw tension resulted. A laryngoscope was used to view the laryngeal folds, and a guarded mare sterile uterine catheter swab (33 in $/ 84 \mathrm{~cm}$ ) was passed through the folds until the tracheal bifurcation; the swab was passed through the guard, and then retracted, followed 
by retraction of the catheter. Anesthesia was reversed with i.v. tolazoline (Tolazine; Lloyd Inc., Shenandoah, IA); a beginning dose of $0.2 \mathrm{mg} / \mathrm{kg}$ was i.v. administered slowly and repeated if necessary until effective, when calves could stand.

\section{Euthanasia and Necropsy}

Calves were euthanized with i.v. sodium pentobarbital (52 mg/kg, Euthasol; Virbac Corp., Forth Worth, TX). Culture for Mycoplasma spp. and a PCR that could differentiate multiple Mycoplasma spp. were performed on postmortem samples of lung, retropharyngeal lymph node, and trachea from each calf. A complete necropsy also was performed to test for other diseases or pathology at the Utah Veterinary Diagnostic Laboratory.

\section{Mycoplasma Culture}

Sand bedding samples were collected weekly from an equal number of superficial (1-2 cm deep into the pile) and deep (15-18 $\mathrm{cm}$ deep into the pile) sites using disposable gloves and a standard sampling-size container and placed in clean, sealable plastic bags. Nasal and ear swab and antemortem tracheal swab samples were placed into CVM transport medium (Hardy Diagnostics, Santa Maria, CA) tubes. Postmortem tissue samples were placed in clean, sealable plastic bags. Samples were held at $4^{\circ} \mathrm{C}$ and shipped within $24 \mathrm{~h}$ to the Dairy Authority Laboratory (Greeley, CO) using a courier service. Bedding samples (an equal mixture of superficial and deep sites) were cultured as reported previously (Justice-Allen et al., 2010), using $1 \mathrm{~g}$ of bedding diluted in $1,000 \mathrm{~mL}$ of sterile water to make an initial 1,000-fold dilution. Three additional 1:10 dilutions were made, resulting in $1: 10^{4}, 1: 10^{5}, 1: 10^{6}$ dilutions. For all dilutions, $50 \mu \mathrm{L}$ of sample was plated and counted to determine colony forming units per gram. Mycoplasma culture was performed on modified Hayflick medium (Hardy Diagnostics, Santa Maria, CA) incubated at $37^{\circ} \mathrm{C}$ with $10 \% \mathrm{CO}_{2}$ and $80 \%$ humidity for $10 \mathrm{~d}$. Plates were examined at 3,7 , and $10 \mathrm{~d}$ under $2 \times$ magnification with confirmation at $4 \times$ magnification, and the number of colonies morphologically consistent with Mycoplasma spp. was counted. The final reported mycoplasma concentration for a sand sample was the mean of all colony forming units per gram determined from all positive dilutions of that sample. Mycoplasma plates that were overgrown with other types of bacteria were defined as contaminated.

\section{DNA Extraction}

Sample preparation and DNA extraction were performed at the Utah Veterinary Diagnostic Laboratory using methods described previously; Mycoplasma bovis had been previously detected in sand samples by PCR as reported earlier (Justice-Allen et al., 2010).

From each tissue sample, a 100-mg piece was minced and then placed in a $2-\mathrm{mL}$ safe-lock tube with a 5 -mm stainless-steel grinding ball with $500 \mu \mathrm{L}$ of tissue lysis buffer (ATL; Qiagen, Valencia, CA) and then homogenized on a shaker for $1 \mathrm{~min}$ at $30 \mathrm{~Hz}$. Then, $40 \mu \mathrm{L}$ of proteinase $\mathrm{K}$ was added and the mixture was incubated for $2 \mathrm{~h}$ at $55^{\circ} \mathrm{C}$. The tissue sample homogenate was then split, with $200 \mu \mathrm{L}$ processed immediately according to the manufacturer's standard protocol, and approximately $300 \mu \mathrm{L}$ stored for retesting.

\section{Mycoplasma Multispecies Real-Time PCR for Tissue Samples}

The reverse primer, MYCP 4 (Eurofins MWG Operon, Huntsville, AL), 5'-GCATCCACCAAAAACTCT-3', was previously developed for a conventional nested protocol (Baird et al., 1999). The forward primer, MYCP 3a (Eurofins MWG Operon), 5'-CATATGTTCTTTGAAAACTG-3', was modified from the forward primer for the conventional nested protocol (Baird et al., 1999) by one of the authors (Trujillo et al., 2009) and overlaps with it. This results in the amplification of a segment of the $16 \mathrm{~S}$ to $23 \mathrm{~S}$ rRNA intergenic spacer and the production of a 210-bp amplicon for M. bovis.

The extracted DNA was diluted to an average concentration of $40 \mu \mathrm{g} / \mathrm{mL}$. The PCR reaction mix consisted of $6.3 \mu \mathrm{L}$ of a SYBR Green master mix (Quanta Biosciences, Gaithersburg, MD), a fluorescent dye that binds to double-stranded DNA during the PCR reaction; $0.5 \mu \mathrm{L}$ of each of the primers (at a concentration of $2.5 \mathrm{pmol} / \mathrm{uL}$; forward, MYCP $3 \mathrm{a}$ and reverse, MYCP 4); and $5.2 \mu \mathrm{L}$ of the diluted extracted DNA. The total volume of the PCR reaction mix was $12.5 \mu \mathrm{L}$. The thermocycling conditions were as follows: 40 cycles of $55^{\circ} \mathrm{C}$ for $2 \mathrm{~min}$ and $72^{\circ} \mathrm{C}$ for 1 min followed by a continuous melt of $95^{\circ} \mathrm{C}$ for $15 \mathrm{~s}, 60^{\circ} \mathrm{C}$ for $1 \mathrm{~min}$, and $95^{\circ} \mathrm{C}$ for 15 $\mathrm{s}$. There was no denaturization step at the beginning of the reaction. Each PCR plate contained positive ( $M$. bovis, ATCC \#25025; American Type Culture Collection, Manassas, VA) and negative (DNA free water) PCR controls, as well as the extraction controls for the group of samples. The testing for each sample was run in triplicate.

\section{Post-PCR Processing}

Amplicon length was compared with an $M$. bovis positive control (ATCC \#25025) using a capillary electrophoresis platform, the 2100 Bioanalyzer (Agilent Technologies Inc., Santa Clara, CA). When an ampli- 
con that differed in length from the $M$. bovis control was identified, a standard electrophoresis gel was run using a molecular-grade, low-melt agarose gel made with Tris-borate-EDTA and GelStar nucleic acid stain (Fisher Scientific, Pittsburgh, PA) at 100 V until good separation of the bands was achieved according to the 100-bp ladder marker. Each DNA band was then cut from the gel, and the DNA was extracted for sequencing using an extraction kit (Qiagen) for DNA in gel. Samples were sequenced on a Sanger DNA sequencing platform, the 3730 DNA analyzer (Applied Biosystems, Foster City, CA) for PCR amplification products at the Center for Integrated Biosystems (Utah State University). The resulting base sequences were subjected to Basic Local Alignment Search Tool (BLAST, http:// blast.ncbi.nlm.nih.gov/Blast.cgi), a search algorithm that compares a sample sequence of nucleotides or AA to sequences that have been identified and stored in the database as part of a specific organism. The sequences stored in the database have been submitted by researchers as being part of various organisms and have been subjected to varying levels of confirmation and analysis.

\section{Statistical Analysis}

Sensitivity for mycoplasma detection was estimated as $25 \%$ for nasal or ear swabs and $75 \%$ for tracheal swabs (Thomas et al., 2002; Marois et al., 2007). Therefore, the probability of a false negative test result was $(1-0.25)$ $=0.75$ for each nasal/ear swab test, and $(1-0.75)=$ 0.25 for each tracheal swab test. For each calf, the probability of being detected positive by at least 1 diagnostic test if they truly became infected with mycoplasma beginning $4 \mathrm{wk}$ after exposure to positive bedding sand was calculated. Because most calves are infected within 1 to 3 wk after exposure to mycoplasma, the assumption that 4 wk of exposure would be necessary before detectable disease resulted was a conservative estimate (Stipkovits et al., 1993; Catry et al., 2008). Sequential probability was used to calculate the probability of all test results for a given calf being falsely negative if the calf became infected (failure to detect a true positive animal at least once). Beginning 4 wk after exposure to positive bedding sand, false-negative probabilities of 0.75 for nasal/ear swab tests were multiplied, and falsenegative probabilities of 0.25 for tracheal swab tests were multiplied. For example, 4 wk after calf 4 was exposed to mycoplasma-positive bedding, he was tested over the next $62 \mathrm{~d}$ with 7 weekly nasal/ear swabs and 2 monthly tracheal swabs; the probability of complete failure to detect infection if present was $(0.75)(0.75)$ $(0.75)(0.25)(0.75)(0.75)(0.75)(0.75)(0.25)=0.008$. The probability of detection by at least 1 positive test result if truly infected with mycoplasma $=(1-0.008)=0.992$ $=99.2 \%$.

\section{RESULTS}

\section{Bedding Sand}

The exposed group sand cultured positive for Mycoplasma spp. during wk 1, 4, 5, 6, 7, and 11 , and negative for the other weeks of the 15 -wk study. The concentrations of mycoplasma in the sand when culture-positive were $14,200,1,400,32,000,600,200$, and $3,000 \mathrm{cfu} / \mathrm{g}$, respectively. The control group sand weekly samples all cultured negative for Mycoplasma spp. Totaled over all exposed group calves, those calves were alive for a total of $385 \mathrm{~d}$ following their first exposures to mycoplasmapositive bedding. The total days that exposed calves were actually bedded on mycoplasma-positive sand was $138 \mathrm{~d}$ (Table 1). It was observed that all of the calves always rooted in the sand when it was added to their hutches, with sand clinging to their muzzles and being visible in the bottom of their water buckets when water was changed at least twice daily.

\section{Antemortem Test Results}

All 94 serum samples had negative results for antibody against $M$. bovis. All 8 tracheal swabs and 41 nasal and ear swabs from exposed calves, as well as all 8 tracheal swabs and 37 nasal and ear swabs from control calves were culture negative for Mycoplasma spp. When counting only samples collected 4 wk after first exposure to mycoplasma in bedding, there were 8 tracheal swabs and 30 nasal and ear swabs from exposed calves, and 8 tracheal swabs and 30 nasal and ear swabs from control calves. The latter numbers, only beginning $4 \mathrm{wk}$ after exposure, were used in the probability of detection of true infections calculations described below and shown in Table 1. For control calves, this was to evaluate the possibility that they may have unintentionally become exposed to mycoplasma at the same time that exposed group calves did by contamination between calf treatment groups despite precautions taken.

\section{Clinical and Necropsy Findings}

Whether calves died or were euthanized, age at death, days elapsed after the calves' first exposure to mycoplasma-positive bedding, days of contact with mycoplasma-positive bedding, and the probability that they would have tested positive at least once for $\mathrm{Myco-}$ plasma spp. if they were infected 4 wk after exposure are shown (Table 1). Except for 3 calves that died or were killed in the first $25 \mathrm{~d}$ of life, the remaining 9 
Table 1. Time of exposure to Mycoplasma bovis: positive bedding sand and probability of detection if infection resulted for 12 naïve dairy calves

\begin{tabular}{lllcccc}
\hline Calf & $\begin{array}{l}\text { Treatment } \\
\text { group }^{1}\end{array}$ & $\begin{array}{l}\text { Died or } \\
\text { euthanized }\end{array}$ & $\begin{array}{c}\text { Age at } \\
\text { death, d }\end{array}$ & $\begin{array}{c}\text { Days after first } \\
\text { exposure to } \\
\text { mycoplasma }\end{array}$ & $\begin{array}{c}\text { Days on } \\
\text { mycoplasma- } \\
\text { positive sand }^{3}\end{array}$ & $\begin{array}{c}\text { Probability of } \\
\text { detection if } \\
\text { infected, }{ }^{4}\end{array}$ \\
\hline 1 & Control & Euthanized & 25 & 0 & 0 & NA \\
2 & Control & Euthanized & 17 & 0 & 0 & NA \\
3 & Control & Euthanized & 113 & 0 & 0 & 99.8 \\
4 & Exposed & Euthanized & 98 & 90 & 35 & 99.2 \\
5 & Exposed & Died & 85 & 80 & 35 & 98.9 \\
6 & Control & Euthanized & 107 & 0 & 0 & 99.8 \\
7 & Control & Euthanized & 76 & 0 & 0 & 99.3 \\
8 & Exposed & Euthanized & 76 & 74 & 25 & 99.3 \\
9 & Exposed & Euthanized & 74 & $74^{5}$ & 4 & NA \\
10 & Exposed & Died & 4 & $4^{5}$ & 14 & 96.5 \\
11 & Exposed & Euthanized & 67 & 63 & 0 & 96.5 \\
12 & Control & Euthanized & 65 & 0 &
\end{tabular}

${ }^{1}$ Treatment group $=$ exposed or control (not exposed) to mycoplasma-positive bedding.

${ }^{2}$ Days after first exposure to mycoplasma $=$ Remaining days the calf lived following the first exposure to mycoplasma-positive bedding, the time during which the calf could have contracted mycoplasma infection.

${ }^{3}$ Days on mycoplasma-positive sand = Actual days the calf was bedded with mycoplasma-positive bedding; bedding was intermittently culture-positive.

${ }^{4}$ Probability of detection if infected $=$ Probability that the calf would have been detected as mycoplasmapositive (by at least one positive test result) if they had become truly infected 4 wh postexposure. This is calculated for control as well as exposed calves to allow for the possibility that they may have somehow become exposed to mycoplasma at the same time that exposed group calves did by contamination between calf treatment groups despite precautions taken. This shows the probability that calves would have been detected with mycoplasma if indeed they became truly infected. NA = Not applicable because the calf did not live until at least $4 \mathrm{wk}$ after exposure.

${ }^{5}$ Calves 9 and 10 arrived the day they were born and were immediately exposed to mycoplasma-positive bedding.

calves had probabilities of between 96.5 and $99.8 \%$ of being detected Mycoplasma spp. positive at least once if they had become truly infected with mycoplasma $4 \mathrm{wk}$ after exposure to positive bedding sand.

Five calves were euthanized because of clinical signs of anorexia, diarrhea, or depression $(\mathrm{n}=3)$ or died suddenly $(\mathrm{n}=2)$ before the end of the 105-d study period. Their ages at death, diagnoses, and necropsy diagnostic results were as follows: calf 1 (control), euthanized at 25 d old, enteric salmonellosis and septicemia, Salmonella spp. isolated from ileum and mesenteric lymph nodes by aerobic culture; calf 2 (control), euthanized at $17 \mathrm{~d}$ old, Escherichia coli septicemia, E. coli isolated by aerobic culture from spleen, liver, meninges, gross and histopathologic lesions of synovitis, enteritis, abomasitis, nephritis, and meningitis; calf 4 (exposed), euthanized at $98 \mathrm{~d}$ old, chronic edematous abomasitis (gross and histopathologic lesions), bovine coronavirus detected by histopathology and immunohistochemistry; calf 5 (exposed), died at 85 d old, E. coli septicemia, cryptosporidiosis, E. coli isolated by aerobic culture from lung and intestine, gross and histopathologic lesions of bacterial enteritis and abomasitis, cryptosporidiosis detected by histopathology and zinc sulfate fecal floatation; calf 10 (exposed), died at $4 \mathrm{~d}$ old, cryptosporidiosis, detected by histopathology, gross and histopathologic lesions of abomasitis. None of the 5 calves had any gross lesions or positive culture or PCR results from the lung, retropharyngeal lymph node, or trachea samples indicating infection with Mycoplasma spp.

The remaining 7 calves (4 controls, 3 exposed) appeared visibly healthy and were euthanized at the end of the study period, aged between 65 and $113 \mathrm{~d}$ old (Table 1). None of the 7 calves had any gross lesions or positive culture or PCR results indicating infection with Mycoplasma spp. In total, all 72 postmortem culture $(\mathrm{n}=36)$ or PCR $(\mathrm{n}=36)$ tests on all 12 calves were negative for Mycoplasma spp.

\section{DISCUSSION}

Bedding sand positive for Mycoplasma bovis showed no evidence of being a source of infection to naïve calves despite exposure over a period of several weeks along with extensive diagnostic testing for mycoplasma in the animals. This has practical implications considering mycoplasma has been found in bedding of dairy herds, including recycled bedding following a manure-separation process in association with mycoplasma mastitis (Justice-Allen et al., 2010). Dairy producers whose herds have been found with mycoplasma mastitis, and whose farms have concurrently had mycoplasma detected in 
bedding samples have asked the question regarding infectivity of the bedding. In this study, mycoplasmapositive bedding was evaluated only for use with young calves, not lactating cows. Other environmental sources of Mycoplasma spp. previously reported on dairy farms include straw and cooling ponds (Bray et al., 1997), but the authors found it much more likely to be found in sand than straw (Justice-Allen et al., 2010).

Limitations of the study were that the bedding sand was positive for only 6 wk of the 15-wk study period, and bedding cultures were performed on samples from the sand pile, but not from individual calf hutches where the sand was used on top of straw. Intermittently positive culture results over time in the pile of sand used in this study had been observed previously as well; the earlier study found that mycoplasma was more likely to be recovered from the sand when the ambient temperature was between 15 and $20^{\circ} \mathrm{C}$, and mycoplasma also was isolated on 2 occasions after the sand pile was moved, including the move to the location for the calf study reported here (Justice-Allen et al., 2010). Mycoplasma recovery from sand in the present study increased following the movement of the sand pile to the calf facility just before the start of the trial, and decreased and later ceased as colder winter temperatures arrived during the late weeks of the project. Nevertheless, the dose of Mycoplasma spp. found in the sand pile when it cultured positive during this experiment ranged between 200 and approximately 30,000 $\mathrm{cfu} / \mathrm{g}$. The infectious dose of $M$. bovis required for nasal transmission of disease to calves has not been demonstrated, but is presumed to be 100 cfu (Rosenbusch, 2003). Therefore, every ounce of mycoplasma-positive bedding sand was likely to contain between 120 and 18,000 times the proposed infectious dose for mycoplasmal calf disease. Conditions in the calf hutches in this study were like those on commercial farms, and failure of mycoplasma-positive sand to be associated with disease was probably not caused by insufficient dose of the organism.

It is beyond the scope of this study to investigate why sand contaminated with mycoplasma did not cause disease. It is possible that inhalation of aerosolized organisms is necessary for infection, and despite the fact that all calves regularly rooted in the sand and covered their muzzles with it, this is not a sufficient vehicle for infection with mycoplasma.

Finding a dairy farm with no evidence of mycoplasma in any preweaned calves was difficult, supporting previous reports that most dairy herds have mycoplasma infections in calves (Springer et al., 1982; ter Laak et al., 1992). Additional evidence that the calves in the study were apparently naïve to mycoplasma included the finding of no antibody against $M$. bovis in any calves for 15 wk. Many calves in most dairy herds have an antibody against $M$. bovis by between 4 and $8 \mathrm{wk}$ of age (Maunsell et al., 2009). In addition, following experimental challenge with $M$. bovis, regardless of whether clinical signs result, calves usually produce an antibody response within 5 d (Nicholas et al., 2002).

\section{CONCLUSIONS}

Further studies regarding transmission of mycoplasma mastitis from contaminated bedding are needed in lactating cows because of the possibility of infection through the teat canals, causing mastitis. However, mycoplasma-positive bedding, at least sand bedding in the conditions of this study, is apparently not a source of infection to nonlactating animals, including preweaned dairy calves.

\section{ACKNOWLEDGMENTS}

This work was funded by grants from the Utah Agricultural Experiment Station, Federal Formula Animal Health Funds, and Utah State University. We appreciate the cooperation of the owners of the source farm for the calves and the help of J. Baldwin and E. Wilson with care and feeding of the calves.

\section{REFERENCES}

Baird, S. C., J. Carman, R. P. Dinsmore, R. L. Walker, and J. K. Collins. 1999. Detection and identification of Mycoplasma from bovine mastitis infections using a nested polymerase chain reaction. J. Vet. Diagn. Invest. 11:432-435.

Bray, D. R., J. K. Shearer, G. A. Donovan, and P. A. Reed. 1997. Approaches to achieving and maintaining a herd free of mycoplasma mastitis. Pages 132-137 in Proc. Natl. Mastitis Counc. Mtg., Albuquerque, NM. Natl. Mastitis Council, Inc., Madison, WI.

Byrne, W. J., R. McCormack, N. Brice, J. Egan, B. Markey, and H. J. Ball. 2001. Isolation of Mycoplasma bovis from bovine clinical samples in the Republic of Ireland. Vet. Rec. 148:331-333.

Catry, B., L. Duchateau, J. Van de Ven, H. Laevens, G. Opsomer, F. Haesebrouk, and A. De Kruif. 2008. Efficacy of metaphylactic florfenicol therapy during natural outbreaks of bovine respiratory disease. J. Vet. Pharmacol. Ther. 31:479-487.

Ghazaei, C. 2006. Mycoplasmal mastitis in dairy cows in the Moghan region of Ardabil State, Iran. J. S. Afr. Vet. Assoc. 77:222-223.

González, R. N., B. M. Jayarao, S. P. Oliver, and P. M. Sears. 1993. Pneumonia, arthritis and mastitis in dairy cows due to Mycoplasma bovis. Pages 178-186 in Proc. Natl. Mastitis Counc. Mtg., Kansas City, MO. Natl. Mastitis Counc., Inc., Arlington, VA.

González, R. N., P. M. Sears, D. J. Wilson, and H. O. Mohammed. 1995. Epidemiology of mycoplasmal bovine mastitis in the state of New York, U.S.A. Pages 68-69 in Proc. 3rd Intl. Dairy Fed. Mastitis Seminar, Tel Aviv, Israel.

González, R. N., and D. J. Wilson. 1997. Where are we on mycoplasmal mastitis? Pages 191-195 in Proc. Cornell 89th Ann. Conf. Vet., Cornell Univ., Ithaca, NY.

Jasper, D. E. 1977. Mycoplasma and mycoplasmal mastitis. J. Am. Vet. Med. Assoc. 170:1167-1172.

Justice-Allen, A., J. Trujillo, R. Corbett, R. Harding, G. Goodell, and D. Wilson. 2010. Survival and replication of Mycoplasma species in recycled bedding sand and association with mastitis on dairy farms in Utah. J. Dairy Sci. 93:192-202. 
Marois, C., J. Le Carrou, M. Kobisch, and A. V. Gautier-Bouchardon. 2007. Isolation of Mycoplasma hyopneumoniae from different sampling sites in experimentally infected and contact SPF piglets. Vet. Microbiol. 120:96-104.

Maunsell, F. P., G. A. Donovan, C. Risco, and M. B. Brown. 2009. Field evaluation of a Mycoplasma bovis bacterin in young dairy calves. Vaccine 27:2781-2788.

Muenster, O. A., E. E. Ose, and T. Matsuoka. 1979. The incidence of Mycoplasma dispar, Ureaplasma and conventional Mycoplasma in the pneumonic calf lung. Can. J. Comp. Med. 43:392-398.

Nicholas, R. A., R. D. Ayling, and L. P. Stipkovits. 2002. An experimental vaccine for calf pneumonia caused by Mycoplasma bovis: Clinical, cultural, serological and pathological findings. Vaccine 20:3569-3575.

Pfützner, H., and K. Sachse. 1996. Mycoplasma bovis as an agent of mastitis, pneumonia, arthritis and genital disorders in cattle. Rev. Sci. Tech. 15:1477-1494.

Rosenbusch, R. F. 2003. Mycoplasma: What we know about mastitis and systemic disease. Pages 8-13 in Proc. Minn. Dairy Health Conf., Univ. Minneapolis, MN.

Springer, W. T., R. W. Fulton, H. V. Hagstad, S. S. Nicholson, and J. D. Garton. 1982. Prevalence of Mycoplasma and Chlamydia in the nasal flora of dairy calves. Vet. Microbiol. 7:351-357.

Stipkovits, L., M. Rady, and R. Glavits. 1993. Mycoplasmal arthritis and meningitis in calves. Acta Vet. Hung. 41:73-88. ter Laak, E. A., J. H. Noordergraaf, and R. P. Dieltjes. 1992. Prevalence of mycoplasmas in the respiratory tracts of pneumonic calves. Zentralbl. Veterinarmed. B. 39:553-562.

Thomas, A., I. Dizier, A. Trolin, J. Mainil, and A. Linden. 2002. Comparison of sampling procedures for isolating pulmonary mycoplasmas in cattle. Vet. Res. Commun. 26:333-339.

Trujillo, J., A. Justice-Allen, T. Morley, and D. Wilson. 2009. SYBR green real-time PCR detection and differentiation assay for $M y$ coplasma species in biological samples. Page 97 in Proc. of the Am. Assoc. Vet. Lab. Diag., San Diego, CA. Am. Assoc. Vet. Lab. Diag., Davis, CA

Wilson, D. J., R. N. González, G. L. Hayes, and P. M. Sears. 1992. Mycoplasma mastitis in dairy herds: History, clinical signs and case reports. Pages 46-51 in Proc. Natl. Mastitis Counc. Mtg., Arlington, VA. Natl. Mastitis Council, Inc., Arlington, VA.

Wilson, D. J., G. Goodell, A. Justice-Allen, and S. T. Smith. 2009 Herd-level prevalence of Mycoplasma spp. mastitis and characteristics of infected dairy herds in Utah as determined by a statewide survey. J. Am. Vet. Med. Assoc. 235:749-754.

Wilson, D. J., R. T. Skirpstunas, J. D. Trujillo, K. B. Cavender, C. V. Bagley, and R. L. Harding. 2007. Unusual history and initial clinical signs of Mycoplasma bovis mastitis and arthritis in firstlactation cows in a closed commercial dairy herd. J. Am. Vet. Med. Assoc. 230:1519-1523. 